\title{
Efficacy of combined modality therapy with sorafenib following hepatic arterial injection chemotherapy and three-dimensional conformal radiotherapy for advanced hepatocellular carcinoma with major vascular invasion
}

\author{
TAKAKO NOMURA $^{1}$, JOJI TANI $^{2}$, AKIHIRO DEGUCHI $^{3}$, MAI NAKAHARA $^{1}$, \\ KYOKO OURA $^{1}$, TOMOKO TADOKORO ${ }^{1}$, KOJI FUJITA ${ }^{1}$, SHIMA MIMURA ${ }^{1}$, TEPPEI SAKAMOTO ${ }^{1}$, \\ ASAHIRO MORISHITA ${ }^{1}$, HIROHITO YONEYAMA ${ }^{1}$, HIDEKI KOBARA ${ }^{1}$, TAKAYUKI SANOMURA ${ }^{4}$, \\ YOSHIHIRO NISHIYAMA ${ }^{4}$, KEIICHI OKANO ${ }^{5}$, YASUYUKI SUZUKI ${ }^{5}$, SHIGEO TAKAHASHI ${ }^{6}$, \\ TORU SHIBATA $^{6}$, KUNIHIKO TSUTSUI ${ }^{1}$, TAKASHI HIMOTO ${ }^{7}$ and TSUTOMU MASAKI $^{1}$
}

\footnotetext{
${ }^{1}$ Department of Gastroenterology and Neurology, Faculty of Medicine, Kagawa University, Kita-gun, Kagawa 761-0793;

${ }^{2}$ Department of Internal Medicine, Yashima General Hospital, Takamatsu, Kagawa 761-0113;

${ }^{3}$ Department of Internal Medicine, Kagawa Rosai Hospital, Marugame, Kagawa 763-8502; Departments of ${ }^{4}$ Radiology and

${ }^{5}$ Gastroenterological Surgery, Faculty of Medicine, Kagawa University; ${ }^{6}$ Department of Radiation Oncology,

Kagawa University Hospital, Kita-gun, Kagawa 761-0793; ${ }^{7}$ Department of Clinical Examination,
}

Faculty of Health Sciences, Kagawa Prefectural University of Health Sciences, Takamatsu, Kagawa 761-0123, Japan

Received December 21, 2018; Accepted August 16, 2019

DOI: $10.3892 / \mathrm{mco} .2019 .1920$

\begin{abstract}
The prognosis of hepatocellular carcinoma (HCC) patients exhibiting macroscopic vascular invasion (MVI) is poor, and the most appropriate treatment approach remains unclear. The current study aimed to investigate the efficacy and safety of sorafenib treatment following chemoradiotherapy for advanced $\mathrm{HCC}$ exhibiting MVI. A newly reported regimen, including 5-fluorouracil and cisplatin therapy (NewFP), plus three-dimensional conformal radiotherapy (3D-CRT) for MVI was used as the initial treatment. Additionally, sorafenib, as a secondary treatment, was administered after NewFP plus 3D-CRT for MVI. The present
\end{abstract}

Correspondence to: Dr Takako Nomura, Department of Gastroenterology and Neurology, Faculty of Medicine, Kagawa University, 1750-1 Ikenobe, Kita-gun, Kagawa 761-0793, Japan E-mail: takako-n@med.kagawa-u.ac.jp

Abbreviations: HCC, hepatocellular carcinoma; MVI, macroscopic vascular invasion; FU, fluorouracil; 3D-CRT, three-dimensional conformal radiotherapy; MST, median survival time; PVTT, portal vein tumor thrombosis; IVCTT, inferior vena cava tumor thrombosis; RT, radiotherapy; TACE, transarterial chemoembolization; HAIC, hepatic arterial infusion chemotherapy; RFA, radiofrequency ablation

Key words: hepatocellular carcinoma, major vascular invasion, sorafenib, hepatic arterial injection chemotherapy, three-dimensional conformal radiotherapy retrospective study enrolled patients with unresectable advanced HCC that was treated with NewFP plus 3D-CRT for MVI between January 2009 and December 2017. In total, 32 HCC patients with MVI were registered. Of these 32 patients, 18 were treated with NewFP plus 3D-CRT for MVI (NewFP + 3D-CRT group) and 14 were treated with sorafenib following NewFP plus 3D-CRT for MVI (sorafenib after NewFP + 3D-CRT group). The study endpoints were overall survival, overall response rate and disease control rate. Clinical factors influencing overall survival were identified using univariate and multivariate analyses. The median survival time in the NewFP $+3 \mathrm{D}-\mathrm{CRT}$ group and sorafenib following NewFP + 3D-CRT group was 6.7 and 49.2 months, respectively $(\mathrm{P}=0.0003)$. For patients with advanced HCC exhibiting MVI, the initial treatment with NewFP plus 3D-CRT for MVI was well tolerated. The administration of sorafenib as the secondary treatment following NewFP plus 3D-CRT for MVI was associated with a significantly higher overall response rate, disease control rate and increased overall survival as compared with the NewFP plus 3D-CRT treatment.

\section{Introduction}

Hepatocellular carcinoma (HCC) is the most common type of liver cancer, and is the third leading cause of cancer deaths worldwide, causing nearly 745,000 deaths each year (1). HCC tends to invade vessels as it progresses and is often associated with macroscopic vascular invasion (MVI). Recent studies found that MVI including portal vein tumor thrombosis (PVTT) and inferior vena cava 
tumor thrombosis (IVCTT) were found in 12.5 to $39.7 \%$ of cases at the time of diagnosis, respectively $(2,3)$. The prognosis of HCC patients showing MVI is extremely poor, and the median survival time (MST) of these patients has been reported to be $2-3$ months $(2,4)$. Sorafenib is the only evidence-based treatment option for patients classified to be at an Advanced stage (C) in the Barcelona Clinic Liver Cancer Staging System (BCLC) (5-7). However, in a pooled analysis of 2 pivotal phase 3 trials, sorafenib prolonged the median survival of these patients by only 47 days compared with placebo (7-9).

Prognosis improvement of advanced HCC is obtained by combined modality therapy according to liver residual function and progress $(10,11)$. With recent technological advances, external beam radiotherapy (RT) could be considered an alternative treatment option for patients with HCC (12). Regarding the role of RT, the effectiveness of three-dimensional conformal radiotherapy (3D-CRT) has been recognized for treating PVTT/IVCTT (13). RT is a better initial therapy option than sorafenib for patients who have advanced unresectable HCC with PVTT (14). A combined treatment consisting of transarterial chemoembolization (TACE) and RT has shown promising radiologic response rates and improved overall survival of HCC with MVI in observational studies $(15,16)$.

Hepatic arterial infusion chemotherapy (HAIC) is used more commonly than systemic chemotherapy, although no survival advantage has been demonstrated. Randomized controlled studies are currently underway to clarify the survival benefit of HAIC. Moreover, various novel systemic chemotherapeutic agents are currently under development in Japan, and further improvements in the treatment outcomes are expected (17). High efficacy and safety of a new combination therapy comprising of cisplatin-lipiodol suspension and 5-FU for HCC with PVTT, referred to as NewFP, has been reported; a high response rate at $86.3 \%$ and a MST of 33 months has been reported with this therapy regimen (18). In patients with advanced HCC and major PVTT, survival was significantly longer in those treated with HAIC combined with RT than it was with sorafenib (19).

This study aimed to investigate the efficacy and safety of NewFP plus 3D-CRT for MVI as the initial treatment and additionally that of sorafenib as the secondary treatment in patients with advanced HCC showing MVI.

\section{Patients and methods}

Patients. This retrospective study enrolled patients from our institute with unresectable advanced HCC who were treated with NewFP plus 3D-CRT for MVI between January 2009 and December 2017. In total, 32 HCC patients with MVI were registered retrospectively. Of these patients, 18 were treated with NewFP plus 3D-CRT for MVI and 14 were treated with sorafenib after NewFP plus 3D-CRT for MVI.

The diagnosis of HCC was made on the basis of the American Association for the Study of Liver Disease (AASLD) guidelines (6). Inclusion criteria were as follows: (i) HCC with PVTT in the first portal branch (Vp3) or in the main portal trunk or contralateral portal branch (Vp4); (ii) HCC with invasion to right or middle or left hepatic vein (Vv2) or IVCTT (Vv3); (iii) removal of all detected tumors is impossible with a sufficient hepatic functional reserve even if thrombectomy using the peel-off technique is considered (20); (iv) Child-Pugh score of 5-8; (v) an Eastern Cooperative Oncology Group (ECOG) performance status (PS) of 0-2 (21); and (vi) no history of radiotherapy to the liver or of sorafenib treatment.

After estimating the response to the initial treatment with NewFP plus 3D-CRT for MVI, sorafenib was given to patients who were not able to undergo curative therapies such as hepatectomy and radiofrequency ablation (RFA) as a secondary treatment.

This study was conducted in accordance with the 1975 Declaration of Helsinki after receiving approval from the institutional review board of the Kagawa University, Kagawa, Japan (approval no. Heisei29-192). The requirement for informed consent from the participants was waived because of the retrospective nature of the study.

Implantation of arterial catheter. An indwelling catheter (5-Fr W and G spiral Catheter; Piolax, Tokyo, Japan) was inserted through the femoral artery, with the distal end extended into the hepatic or gastroduodenal artery with the proximal end connected to the port system (P-U CELSITE PORT; TORAY, Tokyo, Japan), which was implanted subcutaneously. The right gastric, gastroduodenal, and posterior superior pancreaticoduodenal arteries were occluded with microcoils to prevent gastroduodenal ulcers caused by anticancer agents.

Chemotherapy. HAIC involved cisplatin (50 mg fine powder in 5-10 ml lipiodol) and a continuous infusion of 5-FU $(1,500 \mathrm{mg} / 5$ days), referred to as NewFP. On day 1 of treatment, cisplatin with lipiodol was injected through the reservoir catheter, followed by 5-FU $(250 \mathrm{mg})$. Following this, $5-\mathrm{FU}$ $(1,250 \mathrm{mg})$ was continuously infused for 5 days using a balloon pump (SUREFUSER PUMP, Nipro Pharma Corporation, Osaka, Japan). This regimen was administered once per week during the first 2 weeks of admission. Subsequently, a combination of 20-30 mg cisplatin with 2-6 ml lipiodol and 500-1,000 mg 5-FU was infused every 2 weeks at the out-patient department for as long as possible (18). Treatment was discontinued in cases of occurrence of grade 3 or higher adverse effects according to the ECOG classification, with the exception of total bilirubin $>3.0 \mathrm{mg} / \mathrm{dl}$, platelet count $<5 \times 10^{4} / \mu 1$, and leukocyte count $<1,500 / \mu 1$.

Radiotherapy. Planning computed tomography (CT) scans were obtained under free-breathing conditions using scans with a scan time of $3 \mathrm{sec}$ per section. All patients underwent 3D-CRT, planned using a radiation treatment planning system. The gross tumor volume (GTV) consisted of the PVTT or IVCTT. A clinical target volume (CTV) was defined as the GTV with or without the main tumor. If the main tumor existed close to the GTV, the main tumor was included in the CTV when possible. The planning target volume (PTV) consisted of the CTV plus 8-10 mm margins. In principle, a prescribed dose at an isocenter was $50 \mathrm{~Gy}$ in 25 fractions with $2 \mathrm{~Gy}$ per fraction once daily using 6-10 MV photon beams delivered by a linear accelerator. 3D-CRT was started with the first cycle of HAIC. 
Sorafenib treatment. Eligibility criteria for treatment with sorafenib were as follows: (i) unresectable advanced HCC; (ii) no effect of TACE; (iii) no previous sorafenib treatment for the liver tumor; (iv) Child-Pugh class A or B (up to a score of 7 points) hepatic function; (v) an ECOG performance status of 0-2 (21); and (vi) the following laboratory findings: Leukocyte count $>1,500 / \mu 1$, platelet count $>7.5 \times 10^{4} / \mu 1$, and serum hemoglobin level $>8.5 \mathrm{~g} / \mathrm{dl}$. Sorafenib was administered orally as a $400-800 \mathrm{mg}$ dose daily per the discretion of the chief physician. Dose reductions and treatment interruptions were allowed according to drug-related toxicity grades, as recommended.

Assessment of tumor response. To determine the therapeutic effect, baseline tumor measurements were obtained within 1 month before treatment by combining the largest diameters of selected target lesions in each patient, as measured using CT or MRI. CT or MRI was performed 4-6 weeks after the initial treatment cycle and every 2-3 months thereafter. The therapeutic effect was determined according to the best overall response, which was defined by the Modified RECIST (mRECIST) criteria (22). This was as follows: Complete response (CR), the disappearance of any intratumoral arterial enhancement in all target lesions; partial response (PR), at least a 30\% decrease in the sum of diameters of viable (contrast enhancement in the arterial phase) target lesions, taking as reference the baseline sum of the diameters of target lesions; progressive disease (PD), an increase of at least $20 \%$ in the sum of the diameters of viable (enhancing) target lesions, taking as reference the smallest sum of the diameters of viable (enhancing) target lesions recorded since treatment initiation; and stable disease (SD), any case that does not qualify for either PR or PD. Patients who died before their first radiographic assessment were classified as having PD. Data from patients who died without tumor progression were censored. The response rate was defined on the basis of the independent radiologic review as the percentage of patients whose best-response mRECIST rating of CR or PR was maintained for at least 1 month after the first demonstration of such a rating. The disease-control rate was defined on the basis of independent radiologic review as the percentage of patients whose best-response mRECIST rating of CR, PR, or SD was maintained for at least 1 month after the first demonstration of such a rating.

Statistical analysis. All statistical analyses were performed using JMP software (SAS Institute, Inc., Cary, NC, USA), version 13. Baseline patient characteristics were analyzed using the Chi-square test, the Welch's t test, or Fisher's exact probability test. Overall survival rates and progression-free survival (PFS) were calculated using the Kaplan-Meier method and compared using the log-rank test. Changes in hepatic function using Child-Pugh scores before and after 3D-CRT were analyzed using the Wilcoxon signed-rank test. The Cox proportional-hazards model was used to evaluate the interaction between baseline characteristics and overall survival or therapeutic effect. All P values were two-tailed, and values less than 0.05 were considered statistically significant.

\section{Results}

Patient characteristics. Of the $32 \mathrm{HCC}$ patients, 18 were treated with NewFP plus 3D-CRT for MVI (NewFP+3D-CRT group) and 14 were treated with sorafenib after NewFP plus 3D-CRT for MVI (sorafenib after NewFP+3D-CRT group). Comparisons of the clinical features between the two groups are shown in Table I. There were no significant differences in the baseline characteristics between groups.

The NewFP+3D-CRT group $(\mathrm{n}=18)$ included 15 men $(83.3 \%)$ and 3 women (16.7\%), with a mean age of 68 years (Table I). Chronic hepatitis $\mathrm{C}$ virus infection was the predominant cause of $\mathrm{HCC}(\mathrm{n}=7 ; 38.9 \%)$, followed by chronic hepatitis $B$ virus infection $(n=6 ; 33.3 \%)$. Of the 18 patients, 7 patients $(38.8 \%)$ had Child-Pugh class A hepatic function and 11 patients $(61.1 \%)$ had Child-Pugh class B hepatic function. The median maximum tumor diameter was $190 \mathrm{~mm}$. HCC showed portal vein invasion, with 4 patients $(22.2 \%)$ presenting with Vp4 and 14 patients $(77.8 \%)$ with Vp3 type of invasion. Four patients $(22.2 \%)$ had extrahepatic spread (EHS).

The sorafenib after NewFP+3D-CRT group $(n=14)$ included 14 men (100\%), with a mean age of 68.5 years (Table I). Chronic hepatitis $\mathrm{C}$ virus infection was the predominant cause of HCC $(n=9 ; 64.3 \%)$, followed by chronic hepatitis B virus infection $(n=2 ; 14.3 \%)$. Of the 14 patients, 9 patients $(64.2 \%)$ had Child-Pugh class A hepatic function and 5 patients $(35.7 \%)$ had Child-Pugh class B hepatic function. The median maximum tumor diameter was $137 \mathrm{~mm}$. HCC showed portal vein invasion, with 3 patients $(21.4 \%)$ having $\mathrm{Vp} 4$ and 7 patients $(50 \%)$ having Vp3. Two patients (14.3\%) had EHS.

Overall response and efficacy. Table II shows the results at the first radiologic assessment according to the mRECIST. Of the 32 patients treated with NewFP plus 3D-CRT for MVI, $3(9.4 \%), 16(50 \%)$, and $7(21.9 \%)$ patients had CR, PR, and $\mathrm{SD}$, respectively. The overall response rate was $59.4 \%$, and the disease control rate was $81.3 \%$.

Cumulative overall survival curves of patients treated with NewFP plus 3D-CRT for MVI or sorafenib after NewFP plus 3D-CRT for MVI are shown in Fig. 1. The MST was 6.7 months for patients treated with NewFP plus 3D-CRT for MVI and 49.2 months for those treated with sorafenib after NewFP plus 3D-CRT for MVI $(\mathrm{P}=0.0003)$. The PFS in patients showing MVI who were treated either with NewFP plus 3D-CRT or with sorafenib after NewFP plus 3D-CRT are shown in Fig. 2. The median PFS was 4.3 and 6.8 months for patients treated either with NewFP plus 3D-CRT for MVI or those treated with sorafenib after NewFP plus 3D-CRT, respectively $(\mathrm{P}=0.0219)$. Sorafenib, administered as the secondary treatment after NewFP plus 3D-CRT for MVI was associated with a significantly higher overall response rate, disease control rate, and longer overall survival in HCC patients showing MVI.

Factors associated with survival outcomes. The significant prognostic factors for overall survival, according to univariate analysis, were response to initial treatment with NewFP plus 3D-CRT (PR or CR, $\mathrm{P}=0.0479$ ) and the number of tumors $(<4, \mathrm{P}=0.0260)$. Multivariate analysis confirmed that initial treatment with NewFP plus 3D-CRT 
Table I. Patient characteristics $(n=32)$. Baseline patient characteristics were analyzed using the chi-square test, Welch's t test, or Fisher's exact probability test.

\begin{tabular}{|c|c|c|c|}
\hline Variables & NewFP+3D-CRT $(n=18)$ & Sorafenib after NewFP+3D-CRT $(n=14)$ & P-value \\
\hline Age, median (range) & $68(37-83)$ & $68.5(53-80)$ & 0.9130 \\
\hline Sex, n $(\%)$ & & & 0.2379 \\
\hline Male & $15(83.3)$ & $14(100)$ & \\
\hline Female & $3(16.7)$ & $0(0)$ & \\
\hline HBs antigen, $\mathrm{n}(\%)$ & & & 0.2302 \\
\hline Present & $6(33.3)$ & $2(14.3)$ & \\
\hline Absent & $12(66.7)$ & $12(85.7)$ & \\
\hline HCV antibody, n (\%) & & & 0.1641 \\
\hline Present & $7(38.9)$ & $9(64.3)$ & \\
\hline Absent & $11(61.1)$ & $5(35.7)$ & \\
\hline Child-Pugh score, n (\%) & & & 0.0590 \\
\hline 5 & $1(5.6)$ & $1(7.1)$ & \\
\hline 6 & $6(33.3)$ & $8(57.1)$ & \\
\hline 7 & $7(38.9)$ & $5(35.7)$ & \\
\hline 8 & $4(22.2)$ & $0(0)$ & \\
\hline Tumor size, maximum, median (mm) & 190,66 & 137,50 & 0.1587 \\
\hline Number of tumors, $\mathrm{n}(\%)$ & & & 0.7120 \\
\hline$<4$ & $7(38.9)$ & $4(28.6)$ & \\
\hline$\geq 4$ & $11(61.1)$ & $10(71.4)$ & \\
\hline Tumor extent, $\mathrm{n}(\%)$ & & & 0.7178 \\
\hline Unilobar involvement & $12(66.7)$ & $8(57.1)$ & \\
\hline Bilobar involvement & $6(33.3)$ & $6(42.9)$ & \\
\hline $\operatorname{AFP}(\mathrm{ng} / \mathrm{ml}), \mathrm{n}(\%)$ & & & 0.0993 \\
\hline$<1,000$ & $5(27.8)$ & $8(57.1)$ & \\
\hline$\geq 1,000$ & $13(72.2)$ & $6(42.9)$ & \\
\hline DCP median (mAU/ml), n (\%) & & & 0.3893 \\
\hline$<1,000$ & $5(27.8)$ & $6(42.9)$ & \\
\hline$\geq 1,000$ & $13(72.2)$ & $8(57.1)$ & \\
\hline Grade of portal vein invasion, $\mathrm{n}(\%)$ & & & 0.6744 \\
\hline Vp4 & $4(22.2)$ & $3(21.4)$ & \\
\hline Vp3 & $14(77.8)$ & $7(50)$ & \\
\hline Hepatic vein invasion, n (\%) & & & 0.1103 \\
\hline Present & $2(11.1)$ & $4(28.6)$ & \\
\hline Absent & $16(88.9)$ & $10(71.4)$ & \\
\hline Extra-hepatic spread & & & 0.5828 \\
\hline Present & $4(22.2)$ & $2(14.3)$ & \\
\hline Absent & $14(77.8)$ & $12(85.7)$ & \\
\hline
\end{tabular}

NewFP, new 5-fluorouracil and cisplatin therapy; HBs, hepatitis B surface; HCV, hepatitis C virus; AFP, a-fetoprotein; DCP, des-g-carboxy prothrombin; 3D-CRT, three-dimensional conformal radiotherapy.

(PR or CR, hazard ratio, $0.2264 ; 95 \%$ confidence interval, 0.0737-0.6320; $\mathrm{P}=0.0060)$ was an independent factor for overall survival (Table III).

Safety and adverse events. Changes in hepatic function using Child-Pugh scores before and after 3D-CRT are shown in Fig. 3. In surviving cases, no significant hepatic function decline was seen after 3D-CRT. On the other hand, Child-Pugh scores decreased after 3D-CRT; hepatic function improved in several patients.

Serious adverse events such as gastrointestinal bleeding due to radiation gastritis were observed in 3 patients, 1 in the NewFP+3D-CRT group and 2 in the sorafenib after NewFP+3D-CRT group. In all 3 cases, healing occurred using argon plasma coagulation delivered via endoscopy. Treatment-related mortality was not observed in the two groups. 
Table II. Therapeutic effects in all patients $(n=32)$.

\begin{tabular}{lc}
\hline Therapeutic effects & NewFP+3D-CRT $(\mathrm{n}=32)$ \\
\hline CR & 3 \\
PR & 16 \\
SD & 7 \\
PD & 6 \\
ORR (CR+PR), n $(\%)$ & $19(59.4)$ \\
DCR $(C R+P R+S D), n(\%)$ & $26(81.3)$
\end{tabular}

NewFP, new 5-fluorouracil and cisplatin therapy; 3D-CRT, three-dimensional conformal radiotherapy; $\mathrm{CR}$, complete response; $\mathrm{PR}$, partial response; $\mathrm{SD}$, stable disease; $\mathrm{PD}$, progressive disease; ORR, overall response rate; DCR, disease control rate.

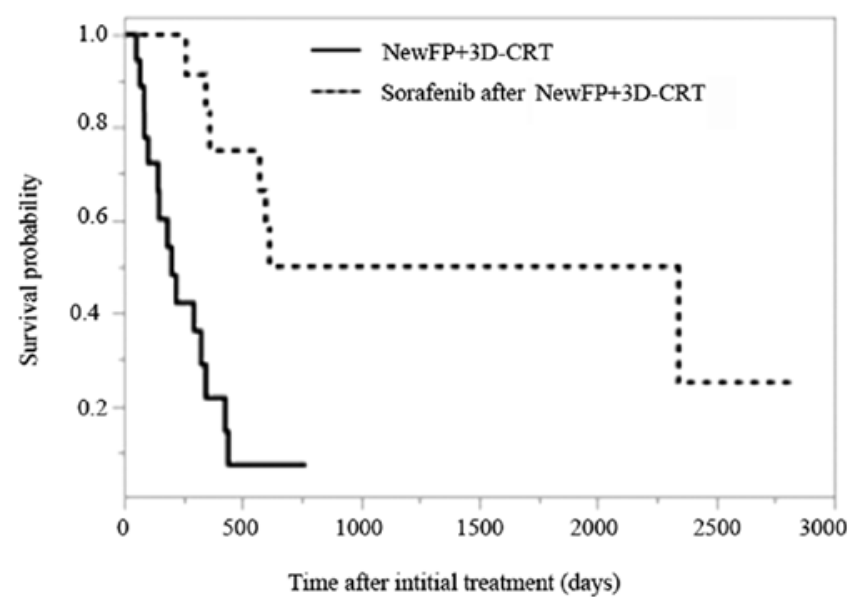

Figure 1. Kaplan-Meier analysis of overall survival in patients treated with NewFP plus 3D-CRT for MVI (solid line; $\mathrm{n}=18$ ) and those treated with sorafenib after NewFP plus 3D-CRT for MVI (dotted line; $\mathrm{n}=14$ ). The median survival times were 6.7 and 49.2 months, respectively. The overall survival rates were compared using the log-rank test. NewFP, new 5-fluorouracil and cisplatin therapy; 3D-CRT, three-dimensional conformal radiotherapy; MVI, macroscopic vascular invasion.

\section{Discussion}

In the present study, for patients with advanced $\mathrm{HCC}$ showing MVI, sorafenib after NewFP plus 3D-CRT for MVI was associated with a significantly higher overall response and disease control rate relative to sorafenib monotherapy. Furthermore, patients administered sorafenib after NewFP plus 3D-CRT for MVI had a good prognosis, with an MST of 49.2 months.

MVI is a prognostic factor for lower overall survival among HCC patients. PVTT causes portal hypertension-related complications such as varix or ascites, and is associated with exacerbating factors such as a larger tumor size, higher tumor grade, and alpha-fetoprotein elevation (23). Because blood from the inferior vena cava flows into the pulmonary vessels, lung metastasis and pulmonary embolism would be expected to be frequent in patients with IVCTT. If the MVI cannot be reduced, it may lead to a decrease in the portal vein blood flow; this may result in a further decline in hepatic function,

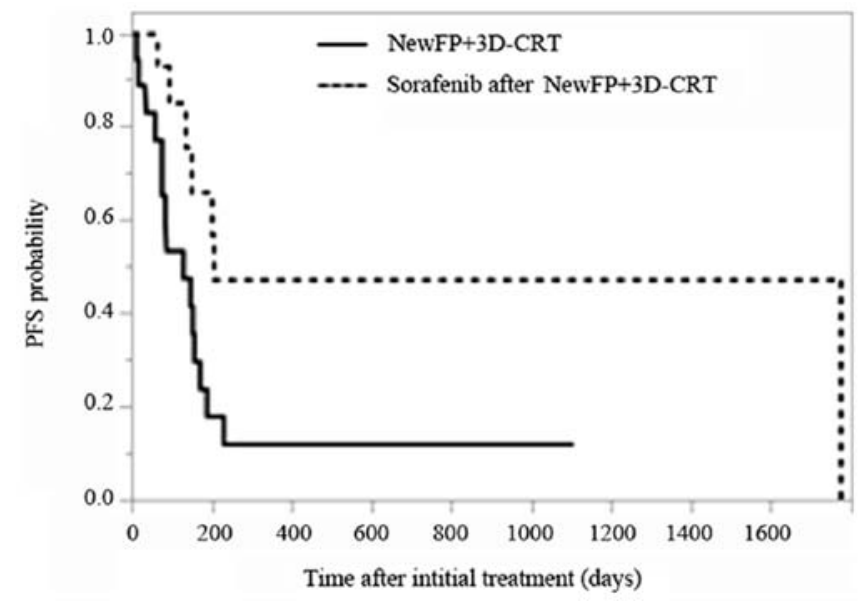

Figure 2. Kaplan-Meier analysis of PFS in patients treated with NewFP plus 3D-CRT for MVI (solid line; $\mathrm{n}=18$ ) and those treated with sorafenib after NewFP plus 3D-CRT for MVI (dotted line; $n=14$ ). The median PFS were 4.3 and 6.8 months, respectively. The PFS rates were compared using the log-rank test. PFS, progression-free survival; NewFP, new 5-fluorouracil and cisplatin therapy; 3D-CRT, three-dimensional conformal radiotherapy; MVI, macroscopic vascular invasion.

increasing the risk of sudden death. Therefore, quick reduction of MVI is important to facilitate subsequent treatment.

Sorafenib is recommended by BCLC guidelines as the first-line therapy for advanced HCC, but its efficacy is limited (24). In the SHARP trial, the MST of patients with HCC showing MVI who were treated with sorafenib was 8.1 months; the incidence of objective responses was low (7). Several studies have reported various combination strategies for HCC showing MVI (25). In a randomized clinical trial for advanced HCC showing MVI, initial treatment with TACE plus RT was well tolerated and conferred an improved progression-free survival, objective response rate, time to progression, and overall survival, compared with sorafenib treatment (26). On the other hand, HAIC combined with RT was associated with a longer MST for HCC with PVTT compared with sorafenib (19). In the present study, immediate therapeutic response was obtained by using NewFP (associated with a high response rate) plus 3D-CRT (associated with a high local control rate) for MVI.

Although regorafenib and lenvatinib can currently be administered as alternatives to sorafenib as molecular target drugs for HCC patients showing MVI, they are limited to Child-Pugh class A hepatic function (27-29). When sorafenib was withdrawn, the introduction of secondary treatments was difficult, due to a decline in hepatic functional reserve, fatigue, and a decline in PS; as recorded in previous reports (30). In the present study, HCC patients showing MVI of Child-Pugh class B hepatic function were safely able to undergo NewFP plus 3D-CRT for MVI as the initial treatment. In addition, several patients in whom hepatic function improved after the initial treatment from Child-Pugh class B to A, were able to receive sorafenib. Furthermore, in the multivariate analysis, initial treatment was extracted as a significant factor associated with overall survival. As the initial treatment, NewFP plus 3D-CRT for MVI was well tolerated and provided a chance for secondary treatment. On the other hand, we compared our study to a previous open label, non-comparative, phase II 
Table III. Factors associated with overall survival. The Cox proportional hazards model was used to evaluate the interaction between baseline characteristics and overall survival or therapeutic effect. Two-tailed values of $\mathrm{P}<0.05$ were considered to indicate a statistically significant result.

\begin{tabular}{|c|c|c|c|}
\hline \multirow[b]{2}{*}{ Variables } & \multirow{2}{*}{$\frac{\text { Univariate analysis }}{\text { P-value }}$} & \multicolumn{2}{|c|}{ Multivariate analysis } \\
\hline & & $\mathrm{HR}(95 \% \mathrm{CI})$ & P-value \\
\hline Age $(<75 / 75 \leq$ years $)$ & 0.3617 & & \\
\hline Child-Pugh (A/B) & 0.1069 & & \\
\hline $\operatorname{AFP}(<1,000 / 1,000 \leq \mathrm{ng} / \mathrm{ml})$ & 0.3891 & & \\
\hline $\mathrm{DCP}(<1,000 / 1,000 \leq \mathrm{mAU} / \mathrm{ml})$ & 0.6205 & & \\
\hline Tumor number $(<4 / 4 \leq)$ & $0.0260^{\mathrm{a}}$ & $0.9731(0.2995-3.0632)$ & 0.9628 \\
\hline Tumor localization (unilobar/bilobar) & 0.3699 & & \\
\hline Vv2-3 (absent/present) & 0.1536 & & \\
\hline Vp3-4 (absent/present) & 0.1536 & & \\
\hline TACE-refractory (abscent/present) & 0.5228 & & \\
\hline Treatment response to NewFP+3D-CRT (PR+CR/SD+PD) & $0.0479^{\mathrm{a}}$ & $0.2264(0.0737-0.6320)$ & $0.0060^{\mathrm{b}}$ \\
\hline
\end{tabular}

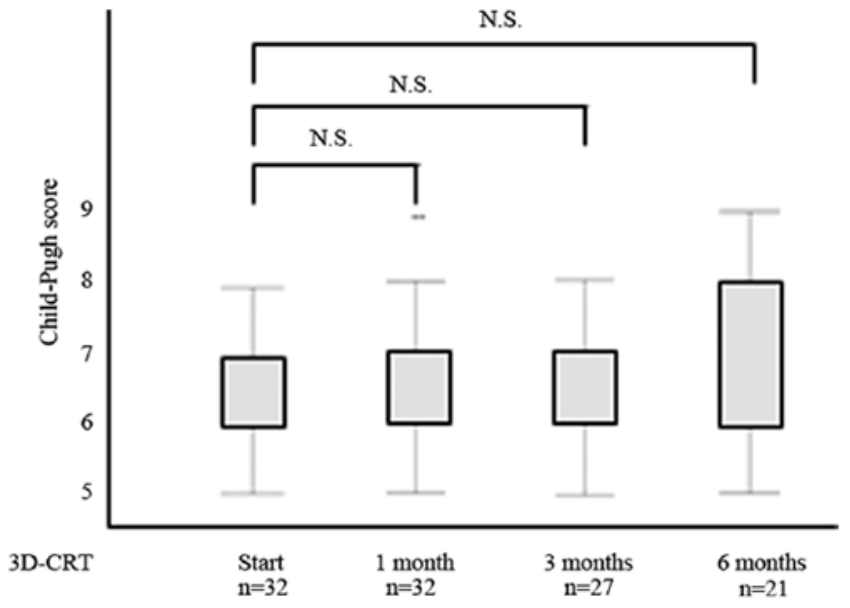

Figure 3. Changes in hepatic function using Child-Pugh scores before and after 3D-CRT. In surviving cases, no significant hepatic functional decline was observed after 3D-CRT. 3D-CRT, three-dimensional conformal radiotherapy. N.S., not statistically significant.

trial in patients with advanced HCC. Patients who received HAIC and were responders were continued on HAIC and were expected to have good prognoses, while the HAIC non-responders were switched to sorafenib (31). The MST of HCC patients showing MVI was 25.4 months in this previous trial. Although there were few differences between our study and the previous trial, the results of sorafenib after NewFP plus 3D-CRT for MVI in our study was superior to that of the previous trial. The reason for our favorable results could be that immediate therapeutic response was obtained by using NewFP (associated with a high response rate) plus 3D-CRT (associated with a high local control rate) for MVI. In addition, avoiding the unnecessary stenosis of hepatic artery by catheter therapy, the reduction of sensitivity to the drug, deterioration of liver function, and appearance of collateral arteries could also explain the favorable results $(32,33)$. We postulated that shifting to the secondary treatment promptly, while maintaining hepatic function, would lead to improvement in prognosis for advanced HCC showing MVI.

Sorafenib, an oral multikinase inhibitor that blocks tumor cell proliferation and angiogenesis, significantly improved overall survival compared with placebo in patients with advanced HCC (7,9). In preclinical studies, sorafenib was shown to exert a synergistic anticancer effect on cisplatin (34). A recent study investigating sorafenib combined with HAIC suggested that combining systemic therapy and regional cytotoxic chemotherapy could enhance antitumor activity (35). Therefore, we speculate that the combination of NewFP and sorafenib synergistically produced an antitumor effect, leading to the higher overall response. Because a considerable proportion of HCC patients showing MVI are unable to receive curative treatment, it is important to explore multimodal strategies for such patients. To the best of our knowledge, NewFP was associated with the longest survival of HCC patients showing MVI in all studies reported so far. No combined modality therapy of NewFP plus 3D-CRT and sorafenib has yet to show a clear survival benefit. The present study is the first reported to show that the combined modality therapy of NewFP plus 3D-CRT and sorafenib is a feasible and promising treatment option for HCC patients showing MVI.

There are several possible limitations to this study. Since this investigation was a retrospective single-center study, the possibility of unintentional selection bias during patient selection cannot be fully excluded. Additionally, the number of cases was small. Therefore, it is necessary to investigate this treatment strategy at multiple centers via a prospective study to confirm our findings.

In conclusion, for patients with advanced HCC showing MVI, the initial treatment with NewFP plus 3D-CRT for MVI was well tolerated, and administration of sorafenib as the secondary treatment after NewFP plus 3D-CRT for MVI was associated 
with a significantly higher overall response rate, disease control rate, and longer overall survival. Our results suggest that a good prognosis could be obtained by performing sorafenib treatment after chemoradiotherapy for advanced HCC showing MVI.

\section{Acknowledgements}

Not applicable.

\section{Funding}

No funding was received.

\section{Availability of data and materials}

The datasets used and analyzed during this study are available from the corresponding author on reasonable request.

\section{Authors' contributions}

TN and TM conceived and designed the study. TN drafted the manuscript. TN, JT, AD, MN, KOu, TT, KF, SM, TSak, AM and HY analyzed and interpreted the data. HK, TSan, YN, KOk, YS, ST, TSh, KT, TH and TM interpreted the data and revised the manuscript critically for important intellectual content. All authors were involved in data interpretation and drafting the manuscript and have read and approved the final version of the manuscript.

\section{Ethics approval and consent to participate}

This study was conducted in accordance with the 1975 Declaration of Helsinki after receiving approval from the institutional review board of the Kagawa University, Kagawa, Japan (approval no. Heisei29-192). The requirement for informed consent from the participants was waived because of the retrospective nature of the study.

\section{Patient consent for publication}

Not applicable.

\section{Competing interests}

The authors declare that they have no competing interests.

\section{References}

1. Ferlay J, Soerjomataram I, Dikshit R, Eser S, Mathers C, Rebelo M, Parkin DM, Forman D and Bray F: Cancer incidence and mortality worldwide: Sources, methods and major patterns in GLOBOCAN 2012. Int J Cancer 136: E359-E386, 2015.

2. Llovet JM, Bustamante J, Castells A, Vilana R, Ayuso Mdel C, Sala M, Brú C, Rodés J and Bruix J: Natural history of untreated nonsurgical hepatocellular carcinoma: Rationale for the design and evaluation of therapeutic trials. Hepatology 29 : 62-67, 1999.

3. Minagawa $\mathbf{M}$ and Makuuchi $\mathrm{M}$ : Treatment of hepatocellular carcinoma accompanied by portal vein tumor thrombus. World J Gastroenterol 12: 7561-7567, 2006.

4. Cabibbo G, Enea M, Attanasio M, Bruix J, Craxi A and Camma C: A meta-analysis of survival rates of untreated patients in randomized clinical trials of hepatocellular carcinoma. Hepatology 51: 1274-1283, 2010.
5. Bruix J, Reig M and Sherman M: Evidence-based diagnosis, staging, and treatment of patients with hepatocellular carcinoma. Gastroenterology 150: 835-853, 2016.

6. Bruix J and Sherman M; American Association for the Study of Liver Diseases: Management of hepatocellular carcinoma: An update. Hepatology 53: 1020-1022, 2011.

7. Llovet JM, Ricci S, Mazzaferro V, Hilgard P, Gane E, Blanc JF, de Oliveira AC, Santoro A, Raoul JL, Forner A, et al: Sorafenib in advanced hepatocellular carcinoma. N Engl J Med 359: 378-390, 2008.

8. Bruix J, Cheng AL, Meinhardt G, Nakajima K, De Sanctis Y and Llovet J: Prognostic factors and predictors of sorafenib benefit in patients with hepatocellular carcinoma: Analysis of two phase III studies. J Hepatol 67: 999-1008, 2017.

9. Cheng AL, Kang YK, Chen Z, Tsao CJ, Qin S, Kim JS, Luo R, Feng J, Ye S, Yang TS, et al: Efficacy and safety of sorafenib in patients in the Asia-Pacific region with advanced hepatocellular carcinoma: A phase III randomised, double-blind, placebo-controlled trial. Lancet Oncol 10: 25-34, 2009.

10. Kudo $\mathrm{M}$ and Ueshima K: Positioning of a molecular-targeted agent, sorafenib, in the treatment algorithm for hepatocellular carcinoma and implication of many complete remission cases in Japan. Oncology 78 (Suppl 1): S154-S166, 2010.

11. Zhao JD, Liu J, Ren ZG, Gu K, Zhou ZH, Li WT, Chen Z, Xu ZY, Liu LM and Jiang GL: Maintenance of Sorafenib following combined therapy of three-dimensional conformal radiation therapy/intensity-modulated radiation therapy and transcatheter arterial chemoembolization in patients with locally advanced hepatocellular carcinoma: A phase I/II study. Radiat Oncol 5: 12, 2010.

12. Citrin DE: Recent developments in radiotherapy. $\mathrm{N}$ Engl J Med 377: 1065-1075, 2017.

13. Huang YJ, Hsu HC, Wang CY, Wang CJ, Chen HC, Huang EY, Fang FM and Lu SN: The treatment responses in cases of radiation therapy to portal vein thrombosis in advanced hepatocellular carcinoma. Int J Radiat Oncol Biol Phys 73: 1155-1163, 2009.

14. Nakazawa T, Hidaka H, Shibuya A, Okuwaki Y, Tanaka Y, Takada J, Minamino $\mathrm{T}$, Watanabe $\mathrm{M}$, Kokubu $\mathrm{S}$ and Koizumi W: Overall survival in response to sorafenib versus radiotherapy in unresectable hepatocellular carcinoma with major portal vein tumor thrombosis: Propensity score analysis. BMC Gastroenterol 14: 84, 2014.

15. Park HC, Yu JI, Cheng JC, Zeng ZC, Hong JH, Wang ML, Kim MS, Chi KH, Liang PC, Lee RC, et al: Consensus for radiotherapy in hepatocellular carcinoma from the 5th Asia-pacific primary liver cancer expert meeting (APPLE 2014): Current practice and future clinical trials. Liver Cancer 5: 162-174, 2016.

16. Wang K, Guo WX, Chen MS, Mao YL, Sun BC, Shi J, Zhang YJ, Meng Y, Yang YF, Cong WM, et al: Multimodality treatment for hepatocellular carcinoma with portal vein tumor thrombus: A large-scale, multicenter, propensity matching score analysis. Medicine (Baltimore) 95: e3015, 2016.

17. Ikeda M, Mitsunaga S, Shimizu S, Ohno I, Takahashi H, Okuyama H, Kuwahara A and Okusaka T: Current status of hepatocellular carcinoma in Japan. Chin Clin Oncol 2: 40, 2013.

18. Nagamatsu H, Hiraki M, Mizukami N, Yoshida H, Iwamoto H, Sumie S, Torimura $\mathrm{T}$ and Sata M: Intra-arterial therapy with cisplatin suspension in lipiodol and 5-fluorouracil for hepatocellular carcinoma with portal vein tumour thrombosis. Aliment Pharmacol Ther 32: 543-550, 2010.

19. Kodama K, Kawaoka T, Aikata H, Uchikawa S, Nishida Y Inagaki Y, Hatooka M, Morio K, Nakahara T, Murakami E, et al: Comparison of outcome of hepatic arterial infusion chemotherapy combined with radiotherapy and sorafenib for advanced hepatocellular carcinoma patients with major portal vein tumor thrombosis. Oncology 94: 215-222, 2018.

20. Inoue Y, Hasegawa K, Ishizawa T, Aoki T, Sano K, Beck Y, Imamura $\mathrm{H}$, Sugawara $\mathrm{Y}$, Kokudo $\mathrm{N}$ and Makuuchi M: Is there any difference in survival according to the portal tumor thrombectomy method in patients with hepatocellular carcinoma? Surgery 145: 9-19, 2009.

21. Therasse P, Arbuck SG, Eisenhauer EA, Wanders J, Kaplan RS, Rubinstein L, Verweij J, Van Glabbeke M, van Oosterom AT, Christian MC and Gwyther SG: New guidelines to evaluate the response to treatment in solid tumors. European organization for research and treatment of cancer, national cancer institute of the United States, national cancer institute of Canada. J Natl Cancer Inst 92: 205-216, 2000. 
22. Lencioni R and Llovet JM: Modified RECIST (mRECIST) assessment for hepatocellular carcinoma. Semin Liver Dis 30: $52-60,2010$.

23. Chan SL, Chong CC, Chan AW, Poon DM and Chok KS: Management of hepatocellular carcinoma with portal vein tumor thrombosis: Review and update at 2016. World J Gastroenterol 22: 7289-7300, 2016.

24. Song DS, Song MJ, Bae SH, Chung WJ, Jang JY, Kim YS, Lee SH, Park JY, Yim HJ, Cho SB, et al: A comparative study between sorafenib and hepatic arterial infusion chemotherapy for advanced hepatocellular carcinoma with portal vein tumor thrombosis. J Gastroenterol 50: 445-454, 2015.

25. Zhu K, Chen J, Lai L Meng X, Zhou B, Huang W, Cai M and Shan H: Hepatocellular carcinoma with portal vein tumor thrombus: Treatment with transarterial chemoembolization combined with sorafenib-a retrospective controlled study. Radiology 272: 284-293, 2014.

26. Yoon SM, Ryoo BY, Lee SJ, Kim JH, Shin JH, An JH, Lee HC and Lim YS: Efficacy and safety of transarterial chemoembolization plus external beam radiotherapy vs sorafenib in hepatocellular carcinoma with macroscopic vascular invasion: A randomized clinical trial. JAMA Oncol 4: 661-669, 2018.

27. Bruix J, Qin S, Merle P, Granito A, Huang YH, Bodoky G, Pracht M, Yokosuka O, Rosmorduc O, Breder V, et al: Regorafenib for patients with hepatocellular carcinoma who progressed on sorafenib treatment (RESORCE): A randomised, double-blind, placebo-controlled, phase 3 trial. Lancet 389: 56-66, 2017.

28. Kudo M: Lenvatinib in advanced hepatocellular carcinoma. Liver Cancer 6: 253-263, 2017

29. Kudo M: A new era of systemic therapy for hepatocellular carcinoma with regorafenib and lenvatinib. Liver Cancer 6: 177-184, 2017.

30. Iavarone M, Cabibbo G, Piscaglia F, Zavaglia C, Grieco A, Villa E, Cammà C and Colombo M; SOFIA (SOraFenib Italian Assessment) study group: Field-practice study of sorafenib therapy for hepatocellular carcinoma: A prospective multicenter study in Italy. Hepatology 54: 2055-2063, 2011.
31. Hatooka M, Kawaoka T, Aikata H, Inagaki Y, Morio K, Nakahara T, Murakami E, Tsuge M, Hiramatsu A, Imamura $\mathrm{M}$, et al: Hepatic arterial infusion chemotherapy followed by sorafenib in patients with advanced hepatocellular carcinoma (HICS55): An open label, non-comparative phase II trial. BMC Cancer 18: 633, 2018.

32. Ikeda M, Mitsunaga S, Shimizu S, Ohno I, Takahashi H, Okuyama H, Kuwahara A, Kondo S, Morizane C, Ueno H, et al: Efficacy of sorafenib in patients with hepatocellular carcinoma refractory to transcatheter arterial chemoembolization. J Gastroenterol 49: 932-940, 2014.

33. Hatooka M, Kawaoka T, Aikata H, Morio K, Kobayashi T, Hiramatsu A, Imamura M, Kawakami Y, Murakami E, Waki K, et al: Comparison of outcome of hepatic arterial infusion chemotherapy and Sorafenib in patients with hepatocellular carcinoma refractory to Transcatheter arterial chemoembolization. Anticancer Res 36: 3523-3529, 2016.

34. Yang Q, Zhang S, Kang M, Dong R and Zhao J: Synergistic growth inhibition by sorafenib and cisplatin in human osteosarcoma cells. Oncol Rep 33: 2537-2544, 2015.

35. Ikeda M, Shimizu S, Sato T, Morimoto M, Kojima Y, Inaba Y, Hagihara A, Kudo M, Nakamori S, Kaneko S, et al: Sorafenib plus hepatic arterial infusion chemotherapy with cisplatin versus sorafenib for advanced hepatocellular carcinoma: Randomized phase II trial. Ann Oncol 27: 2090-2096, 2016.

This work is licensed under a Creative Commons Attribution-NonCommercial-NoDerivatives 4.0 International (CC BY-NC-ND 4.0) License. 